\title{
Delayed Parkinson's Disease Diagnosis among African-Americans: The Role of Reporting of Disability
}

\author{
Nabila Dahodwala ${ }^{a-c}$ Jason Karlawish ${ }^{b, d}$ Andrew Siderowf ${ }^{a-c}$ John E. Duda ${ }^{a, c}$ \\ David S. Mandell ${ }^{\text {b, e }}$ \\ aParkinson's Disease Research, Education and Clinical Center, Philadelphia VA Medical Center, and \\ ${ }^{b}$ Leonard Davis Institute of Health Economics and Departments of ${ }^{\mathrm{C}}$ Neurology, ${ }^{\mathrm{d}}$ Medicine and \\ e Psychiatry, University of Pennsylvania, Philadelphia, Pa., USA
}

\section{Key Words}

African-American · Delay · Diagnosis $\cdot$ Health services •

Parkinsonism $\cdot$ Race

\begin{abstract}
Background/Aims: Racial differences in the observed prevalence of Parkinson's disease (PD) may be due to delayed diagnosis among African-Americans. We sought to compare the stage at which African-American and white PD patients present for healthcare, and determine whether perception of disability accounts for racial differences. Methods: Using records of veterans with newly diagnosed PD at the Philadelphia Veterans Affairs Medical Center, we calculated differences in reporting of symptoms as the difference in z-scores on the Unified Parkinson Disease Rating Scale part 2 (disability) and part 3 (motor impairment). Ordinal logistic regression was used to determine predictors of stage at diagnosis. Results: African-American $(n=16)$ and white $(n=58)$ veterans with a mean age of 70.1 years were identified. AfricanAmericans presented at a later PD stage than whites (median Hoehn + Yahr stage 2.5 vs. 2.0, $p=0.02$ ) and were more likely to under-report disability relative to motor impairment (81 vs. $40 \%, p<0.01$ ). Multivariate analysis showed that underreporting of disability accounted for much of the effect of
\end{abstract}

race on stage of diagnosis. Conclusions: Under-reporting of disability among African-Americans may account for later stages of PD diagnosis than whites. This study begins to explain the mechanisms underlying observed racial disparities in PD.

Copyright $\odot 2011$ S. Karger AG, Basel

\section{Introduction}

The diagnosis of Parkinson's disease (PD) is based on a physician's impression after interviewing and examining a patient. The diagnosis of PD, therefore, relies partly upon a patient's self-report of movement-related disability. Unfortunately, epidemiological studies have shown that between 12 and 78\% of PD cases are undiagnosed [1-6], which may occur if individuals under-report relevant symptoms.

Self-reported disability does not always correlate with clinician-observed impairment [7-9]. Although one study found no patient characteristics associated with concordance among self-reported disability, caregiver report and independent observation [10], another study suggests that earlier stages of $\mathrm{PD}$, shorter duration of $\mathrm{PD}$, and living with a spouse are associated with patients' un-

Nabila Dahodwala, MD, MS

Parkinson's Disease and Movement Disorders Center

330 S. 9 th St., 2nd floor

Philadelphia, PA 19107 (USA)

Tel. +1 215829 6708, E-Mail dahodwan@mail.med.upenn.edu 
der-reporting symptoms [7]. In addition, cognitive dysfunction may contribute to impaired awareness of symptoms in PD [9]. Unfortunately, no study has examined the role of under-reporting of symptoms in missed or delayed diagnosis of PD.

Researchers have described racial disparities in PD diagnosis [11-13]. The majority of these studies suggest that African-Americans are less likely to be diagnosed with PD than whites. One community-based door-to-door study found that African-Americans were at a higher risk of missed diagnosis of PD than whites [6]. This suggests that the relatively lower observed prevalence of $\mathrm{PD}$ among African-Americans is due to missed diagnosis rather than biological differences.

We hypothesize that if racial differences in PD diagnosis are due to delays in diagnosis among AfricanAmericans, then they will present for care at a later stage than whites. We also hypothesize that this delayed diagnosis is due to under-reporting of disability (alteration in the capacity to function in personal, social or occupational demands) relative to clinician-observed impairment (alteration in health status).

\section{Methods and Materials}

The Philadelphia Veteran's Affairs (VA) Medical Center institutional review board approved the study with a waiver of informed consent.

Data Source and Sample

From the electronic health record at the Philadelphia VA Medical Center, we abstracted initial visits potentially related to parkinsonism (ICD-coded idiopathic PD, atypical parkinsonism, secondary parkinsonism and tremor) from January 2001 to September 2010. The VA is a government-funded national system of healthcare facilities that provides comprehensive physical and mental health services to over 5 million veterans of the US military service; approximately $13 \%$ are African-American [14]. The patients were seen in the Parkinson's Disease Research, Education and Clinical Center which is 1 of 6 centers throughout the nationwide VA system for the care of veterans with PD and related disorders. Patients are referred to the movement disorder neurologists in the center from general neurologists as well as directly from primary care providers. Charts were reviewed to determine cases of newly diagnosed possible PD using established criteria [15].

\section{Variables}

Self-reported disability and physician-reported impairment were measured using the Unified Parkinson's Disease Rating Scale (UPDRS), a validated 31-item PD scale that assesses: mentation, behavior and mood (part 1); activities of daily living/disability (part 2); motor examination/impairment (part 3). Responses range from 0 (normal) to 4 (severely disabled/impaired). The

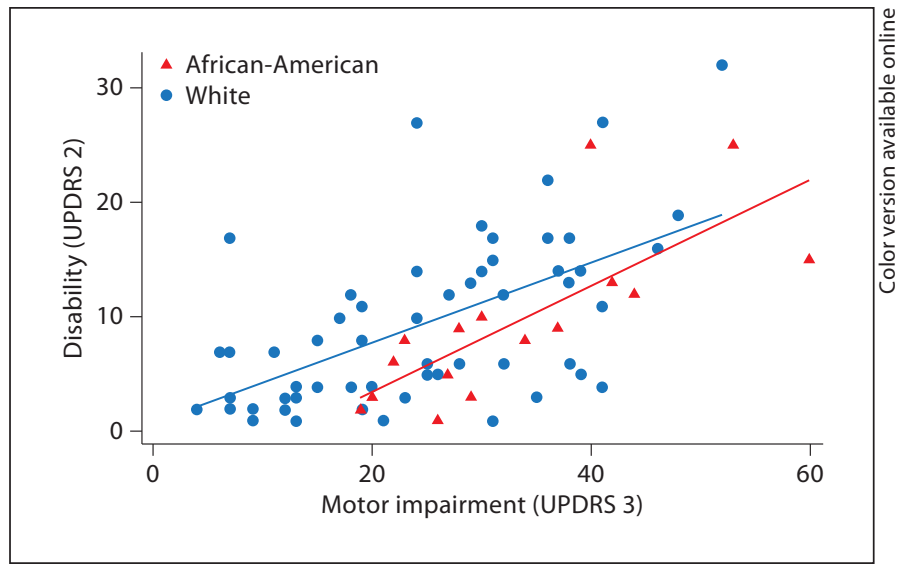

Fig. 1. Scores on self-reported disability scale (UPDRS 2) versus motor impairment (UPDRS 3) by race.

items of the UPDRS part 2 were developed to assess functional symptoms of PD. Prior work has supported the use of the UDPRS part 2 as a self-report measure, and has shown reliability between patient, caregiver and clinician ratings $[16,17]$. All patients completed the activities of daily living/disability (UPDRS 2) scale prior to their visit. A movement-disorders-trained board-certified neurologist completed the motor examination/impairment (UPDRS 3) scale. Additional data abstracted from the medical record included age, sex, race, years of education, duration of symptoms, symptoms of depression (UPDRS 1), assessment of modified Hoehn and Yahr $(\mathrm{H}+\mathrm{Y})$ stage and cognitive assessment adjusted for education (using either the Montreal Cognitive Assessment, Mini-Mental Status Examination, MMSE, or St. Louis University Mental Status examination).

Data Analysis

We compared the $\mathrm{H}+\mathrm{Y}$ stage at which patients presented for care by race using the Wilcoxon rank-sum test. Next, we converted scores on the self-reported disability scale (UPDRS 2) and the clinician-observed motor impairment (UPDRS 3) to z-scores, and then calculated the degree of reporting of symptoms relative to motor impairment as the difference between the z-scores. Underreporters were defined as individuals whose $\mathrm{z}$-score difference (standardized UPDRS 2 - standardized UPDRS 3) was less than zero. To predict what sociodemographic factors explain the stage at PD diagnosis, we created two ordinal logistic regression models: model 1 examined sociodemographic factors without the measure of under-reporting of disability symptoms relative to motor impairment: $\log$ [odds(one unit increase in $\mathrm{H}+\mathrm{Y}$ stage) $]=$ $B_{0}+B_{1} \times$ race $+B_{2} \times$ age $+B_{3} \times$ education; model 2 added the measure of under-reporting of disability symptoms relative to motor impairment: $\log$ [odds(one unit increase in $\mathrm{H}+\mathrm{Y}$ stage) $]=$ $B_{0}+B_{1} \times$ race $+B_{2} \times$ age $+B_{3} \times$ education $+B_{4} \times$ under-report of symptoms). To measure whether under-reporting of disability was an independent predictor of stage at diagnosis, we used the likelihood ratio test to assess the goodness-of-fit of the logistic regression models. 
Table 1. Demographic and clinical characteristics of newly diagnosed veterans with PD

\begin{tabular}{|c|c|c|c|}
\hline & $\begin{array}{l}\text { African-American } \\
(\mathrm{n}=16)\end{array}$ & $\begin{array}{l}\text { White } \\
(\mathrm{n}=58)\end{array}$ & $\mathrm{p}$ value \\
\hline Age, years & $70.8 \pm 9.5$ & $70.0 \pm 10.3$ & $0.96^{\mathrm{a}}$ \\
\hline Males, \% & $93.8(\mathrm{n}=15)$ & $98.3(\mathrm{n}=57)$ & $0.39^{\mathrm{b}}$ \\
\hline Education, years ${ }^{1}$ & $11.9 \pm 1.5$ & $12.7 \pm 2.6$ & $0.34^{\mathrm{a}}$ \\
\hline Duration of symptoms prior to initial visit, months ${ }^{2}$ & $12(1-84)$ & $12(1-240)$ & $0.50^{\mathrm{c}}$ \\
\hline Total UPDRS & $40(21-85)$ & $32(7-100)$ & $0.11^{\mathrm{c}}$ \\
\hline UPDRS part 2 - disability & $8.5(1-25)$ & $7(1-32)$ & $0.82^{\mathrm{c}}$ \\
\hline UPDRS part 3 - motor impairment & $29.5(19-60)$ & $24(4-52)$ & $0.02^{\mathrm{c}}$ \\
\hline \multicolumn{4}{|l|}{$\mathrm{H}+\mathrm{Y}$ stage, $\%$} \\
\hline 1 & $0(\mathrm{n}=0)$ & $25.9(n=15)$ & \\
\hline 1.5 & $0(\mathrm{n}=0)$ & $6.9(\mathrm{n}=4)$ & \\
\hline 2 & $43.7(\mathrm{n}=7)$ & $32.8(\mathrm{n}=19)$ & \\
\hline 2.5 & $25.0(\mathrm{n}=4)$ & $13.8(\mathrm{n}=8)$ & \\
\hline 3 & $25.0(n=4)$ & $19.0(\mathrm{n}=11)$ & \\
\hline 4 & $6.3(\mathrm{n}=1)$ & $1.7(\mathrm{n}=1)$ & \\
\hline 5 & $0(\mathrm{n}=0)$ & $0(\mathrm{n}=0)$ & \\
\hline Median $\mathrm{H}+\mathrm{Y}$ stage & 2.5 & 2 & $0.02^{\mathrm{c}}$ \\
\hline Underreported disability symptoms relative to motor impairment, $\%^{3}$ & $81.3(\mathrm{n}=13)$ & $39.7(\mathrm{n}=23)$ & $<0.01^{\mathrm{b}}$ \\
\hline Tremor, $\%$ & $81.3(\mathrm{n}=13)$ & $93.1(\mathrm{n}=54)$ & $0.17^{\mathrm{b}}$ \\
\hline Depression item, score & $0(0-3)$ & $0(0-4)$ & $0.84^{\mathrm{c}}$ \\
\hline Cognitive impairment (by MoCA, MMSE or SLUMS), $\%{ }^{4}$ & $61.5(\mathrm{n}=8)$ & $46.9(\mathrm{n}=23)$ & $0.35^{\mathrm{d}}$ \\
\hline
\end{tabular}

Where indicated, data presented as means \pm SD or medians (ranges). MoCA $=$ Montreal Cognitive Assessment; SLUMS $=$ St. Louis University Mental Status examination. ${ }^{1} 14$ missing; ${ }^{2} 3$ missing; ${ }^{3}$ calculated as: UPDRS 2 z-score - UDPRS 3 z-score; ${ }^{4} 12$ miss-

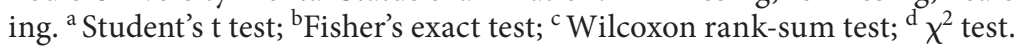

\section{Results}

Seventy-four newly diagnosed cases of idiopathic PD were identified. Of these patients, $22 \%(n=16)$ were African-American and $78 \%(\mathrm{n}=58)$ were white. The majority $(97 \% ; n=72)$ were male. African-Americans with newly diagnosed PD had, on average, greater motor impairment (UPDRS 3 score 29.5 vs. 24; $\mathrm{p}=0.02$ ), later $\mathrm{H}+\mathrm{Y}$ stage (2.5 vs. $2.0 ; \mathrm{p}=0.02)$ and lower self-report of disability relative to motor examination ( 81.3 vs. $39.7 \%$; $\mathrm{p}<$ 0.01 ) (table 1). There was no significant difference in the presence of rest tremor, depression or cognitive impairment between African-Americans and whites.

Disability (UPDRS 2) and motor impairment (UPDRS 3) scores were linearly correlated; however, the relationship differed by race (fig. 1). At the same level of motor impairment, African-Americans reported less disability. The odds of reporting any disability after controlling for difference in motor examination was significantly lower among African-Americans than whites (OR 0.35, 95\% CI $0.13-0.97, \mathrm{p}=0.04)$.
After controlling for age and education, African-American race was strongly associated with later stage at diagnosis, with an increased OR of 3.32 (95\% CI 1.01-10.93, $\mathrm{p}=0.05)$ of presenting 1 stage later than whites. In this first model, age was significantly associated with later stage of diagnosis (OR 1.06, 95\% CI 1.01-1.11, p = 0.03) while education was not (OR 0.85, 95\% CI 0.69-1.05, p = 0.13).

The second model included the variable indicating whether individuals under-reported disability relative to motor impairment. In this model, the magnitude of the association between race and stage at diagnosis decreased by $30 \%$ and the OR was no longer statistically significant (OR 2.34, 95\% CI 0.67-8.19, p = 0.18). Individuals who under-reported symptoms had 2.98 times the odds $(95 \%$ CI $1.03-8.60, \mathrm{p}=0.04$ ) of presenting for care 1 stage later than those who did not under-report symptoms. Older age, but not years of education, remained associated with later state at diagnosis (OR 1.05, 95\% CI 1.00-1.10, p = 0.04 for older age and OR $0.87,95 \%$ CI $0.70-1.08, \mathrm{p}=0.22$ for years of education). The likelihood ratio test $\chi^{2}$ statistic for the goodness-of-fit of the regression model was $4.24(\mathrm{p}=0.04)$. 


\section{Discussion}

In this sample of veterans receiving a first diagnosis of $\mathrm{PD}$, African-Americans were diagnosed at a later stage than whites. African-Americans were also more likely to under-report symptoms of parkinsonism-related disability relative to motor examination. This under-reporting of symptoms accounted for a significant portion of the racial difference in stage at diagnosis.

Prior studies have described racial differences in the prevalence and incidence of PD [11-13]. This study is the first to suggest a mechanism of these observed disparities in diagnosis, that is, racial differences in perceived disability. There are several potential reasons for these differences.

One possibility is related to racial or ethnic differences in attitudes and beliefs about aging [18]. For example, in a previous study, depressed older adults who attributed depressive symptoms to old age were less likely to believe it was important to discuss them with a doctor [19]. The under-reporting of disability related to parkinsonism may be associated with the belief that these symptoms are a normal part of aging. Consequently, any misattribution of symptoms to normal aging would lead to delays in PD diagnosis. Alternatively, cognitive dysfunction in PD may contribute to impaired recognition of symptoms [9]. However, we observed that while cognitive impairment was weakly associated with under-reporting of symptoms, it was not associated with race.

Causes of racial disparities in other disease conditions, such as cardiovascular disease, may also apply to PD. Some studies indicate that minority patients are less likely to trust their physician and are less active participants in their care, which can interfere with effective patientphysician communication [14]. It is possible that AfricanAmericans report less disability because of decreased trust, or are less vocal about expressing concerns. Additionally, limited social support and resources among African-Americans could translate into less assistance with illness recognition and medical decision-making [14].

Several study limitations should be noted. Findings from patients referred to a specialty center may not generalize to the broader population. They may represent atypical case presentations that require specialty consultation. Alternatively, patients referred to a specialty center may be more aware of parkinsonism and the importance of seeking care. Related, the VA represents a unique population with fewer women and greater co-morbidity than the general population. However, it also represents a setting where access to services are equal across race and ethnicity, and is thus ideal to study the causes of racial disparities [14]. Furthermore, prior evidence does not suggest that referral to specialty centers or neurological care for PD differs by race $[20,21]$.

Second, we identified potential cases of parkinsonism by administrative codes, and thus would have missed any cases which were misclassified. If the missed cases were systematically different by race, this would have biased our findings. Lastly, the small sample and extent of missing data may have led to decreases in the ability to detect a significant association between other demographic or clinical factors and race or delays in diagnosis.

\section{Conclusions}

This study has several implications in understanding the mechanisms of racial disparities in PD. Delayed diagnosis of PD among African-Americans may help explain racial differences in studies of $\mathrm{PD}$ prevalence that rely on self-report screens or administrative records for case ascertainment. Furthermore, these differences may be due to under-reporting of disability, as this explained a substantial proportion of the delayed diagnosis by race. As the root causes of racial disparities in PD are uncovered and explored, subsequent targeted interventions can be developed to help reduce them.

\section{Acknowledgment}

We thank Eileen Hummel, BSN, for her assistance with data collection.

\section{Funding}

This study was funded by a Pilot Project Award from the Philadelphia VAMC Parkinson's Disease Research, Education and Clinical Center. N.D. is supported by NIH grant K23 AG034236.

References

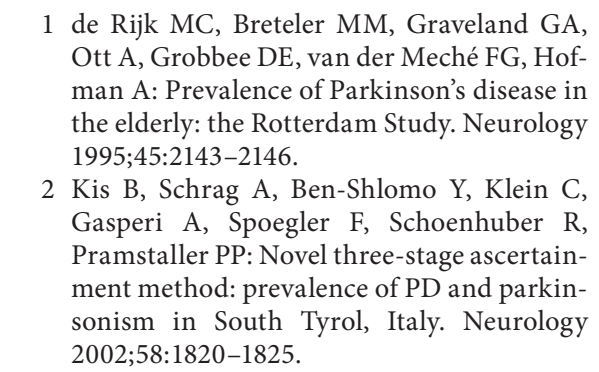

Neuroepidemiology 2011;36:150-154 
3 Tison F, Dartigues JF, Dubes L, Zuber M, Alperovitch A, Henry P: Prevalence of Parkinson's disease in the elderly: a population study in Gironde, France. Acta Neurol Scand 1994;90:111-115.

4 Melcon MO, Anderson DW, Vergara RH, Rocca WA: Prevalence of Parkinson's disease in Junin, Buenos Aires province, Argentina. Mov Disord 1997;12:197-205.

5 Dotchin C, Msuya O, Kissima J, Massawe J, Mhina A, Moshy A, Aris E, Jusabani A, Whiting D, Masuki G, Walker R: The prevalence of Parkinson's disease in rural Tanzania. Mov Disord 2008;23:1567-1572.

$\checkmark 6$ Schoenberg BS, Anderson DW, Haerer AF: Prevalence of Parkinson's disease in the biracial population of Copiah county, Mississippi. Neurology 1985;35:841-845.

7 Shulman LM, Pretzer-Aboff I, Anderson KE, Stevenson R, Vaughan CG, Gruber-Baldini AL, Reich SG, Weiner WJ: Subjective report versus objective measurement of activities of daily living in Parkinson's disease. Mov Disord 2006;21:794-799.

$>8$ Leritz E, Loftis C, Crucian G, Friedman W, Bowers D: Self-awareness of deficits in Parkinson disease. Clin Neuropsychol 2004;18: 352-361.
9 Seltzer B, Vasterling JJ, Mathias CW, Brennan A: Clinical and neuropsychological correlates of impaired awareness of deficits in Alzheimer disease and Parkinson disease: a comparative study. Neuropsychiatry Neuropsychol Behav Neurol 2001; 14:122-129.

10 Brown RG, MacCarthy B, Jahanshahi M, Marsden CD: Accuracy of self-reported disability in patients with parkinsonism. Arch Neurol 1989;46:955-959.

11 McInerney-Leo A, Gwinn-Hardy K, Nussbaum RL: Prevalence of Parkinson's disease in populations of African ancestry: a review. J Nat Med Assoc 2004;96:974-979.

12 Dahodwala N, Siderowf A, Xie M, Noll E, Stern MB, Mandell DS: Racial differences in the diagnosis of Parkinson's disease. Mov Disord 2009;24:1200-1205.

13 Wright Willis A, Evanoff BA, Lian M, Criswell SR, Racette BA: Geographic and ethnic variation in parkinson disease: a population-based study of US medicare beneficiaries. Neuroepidemiology 2010;34:143151.

14 Saha S, Freeman M, Toure J, Tippens KM, Weeks C, Ibrahim S: Racial and ethnic disparities in the VA health care system: a systematic review. J Gen Intern Med 2008;23: 654-671.

15 Gelb DJ, Oliver E, Gilman S: Diagnostic criteria for Parkinson disease. Arch Neurol 1999;56:33-39.
16 Louis ED, Lynch T, Marder K, Fahn S: Reliability of patient completion of the historial section of the unified Parkinson's disease rating scale. Mov Disord 1996;11:185-192.

17 Martinez-Martin P, Benito-Leon J, Alonso F, Catalan MJ, Pondal M, Tobias A, Zamarbide I: Patients', doctors', and caregivers' assessment of disability using the UPDRS-ADL section: are these ratings interchangeable? Mov Disord 2003;18:985-992.

18 Sarkisian CA, Shunkwiler SM, Aguilar I, Moore AA: Ethnic differences in expectations for aging among older adults. J Am Geriatr Soc 2006;54:1277-1282.

19 Sarkisian CA, Lee-Henderson MH, Mangione CM: Do depressed older adults who attribute depression to 'old age' believe it is important to seek care? J Gen Intern Med 2003; 18:1001-1005.

20 Dahodwala N, Xie M, Noll E, Siderowf A, Mandell DS: Treatment disparities in Parkinson's disease. Ann Neurol 2009;66:142145.

-21 Rybicki BA, Johnson CC, Gorell JM: Demographic differences in referral rates to neurologists of patients with suspected Parkinson's disease: implications for case-control study design. Neuroepidemiology 1995;14: $72-81$. 